\title{
Accounting for exotic matter and the extreme radial tension in Morris-Thorne wormholes of embedding class one
}

\author{
Peter K. F. Kuhfittig ${ }^{\mathrm{a}}$ \\ Department of Mathematics, Milwaukee School of Engineering, Milwaukee, WI 53202-3109, USA
}

Received: 17 June 2021 / Accepted: 25 July 2021 / Published online: 30 August 2021

(C) The Author(s) 2021

\begin{abstract}
The embedding of a curved spacetime in a higherdimensional flat spacetime has continued to be a topic of interest in the general theory of relativity, as exemplified by the induced-matter theory. This paper deals with spacetimes of embedding class one, i.e., spacetimes that can be embedded in a five-dimensional flat spacetime. Einstein's theory allows the fifth dimension to be either spacelike or timelike. By assuming the latter, this paper addresses two fundamental issues concerning Morris-Thorne wormholes, the origin of exotic matter and the frequently inexplicable enormous radial tension at the throat.
\end{abstract}

\section{Introduction}

Wormholes are handles or tunnels in spacetime connecting widely separated regions of our Universe or different universes in a multiverse. Apart from some forerunners, macroscopic traversable wormholes were first studied in detail by Morris and Thorne [1] in 1988. They had proposed the following static and spherically symmetric line element for a wormhole spacetime:

$d s^{2}=-e^{\nu(r)} d t^{2}+e^{\lambda(r)} d r^{2}+r^{2}\left(d \theta^{2}+\sin ^{2} \theta d \phi^{2}\right)$,

where

$e^{\lambda(r)}=\frac{1}{1-\frac{b(r)}{r}}$.

(We are using units in which $c=G=1$; the only exceptions are Eqs. (8) and (9) since these involve numerical calculations.) Here $v=v(r)$ is called the redshift function, which must be everywhere finite to prevent an event horizon. The function $b=b(r)$ is called the shape function since it determines the spatial shape of the wormhole when viewed, for

a e-mail: kuhfitti@msoe.edu (corresponding author) example, in an embedding diagram [1]. The spherical surface $r=r_{0}$ is called the throat of the wormhole, where $b\left(r_{0}\right)=r_{0}$. The shape function must also meet the following requirements: $b^{\prime}\left(r_{0}\right)<1$, called the flare-out condition, and $b(r)<r$ for $r>r_{0}$. A final requirement is asymptotic flatness: $\lim _{r \rightarrow \infty} v(r)=0$ and $\lim _{r \rightarrow \infty} b(r) / r=0$.

The flare-out condition can only be met by violating the null energy condition (NEC), $T_{\alpha \beta} k^{\alpha} k^{\beta} \geq 0$, for all null vectors $k^{\alpha}$, where $T_{\alpha \beta}$ is the energy-momentum tensor. Matter that violates the NEC is called "exotic" in Ref. [1]. For the outgoing null vector $(1,1,0,0)$, the violation becomes

$T_{\alpha \beta} k^{\alpha} k^{\beta}=\rho+p_{r}<0$.

Here $T_{t}^{t}=-\rho$ is the energy density, $T_{r}^{r}=p_{r}$ is the radial pressure, and $T_{\theta}^{\theta}=T_{\phi}^{\phi}=p_{t}$ is the lateral (transverse) pressure.

Before continuing, let us list the Einstein field equations:

$$
\begin{aligned}
8 \pi \rho & =e^{-\lambda}\left[\frac{\lambda^{\prime}}{r}-\frac{1}{r^{2}}\right]+\frac{1}{r^{2}}, \\
8 \pi p_{r} & =e^{-\lambda}\left[\frac{1}{r^{2}}+\frac{v^{\prime}}{r}\right]-\frac{1}{r^{2}},
\end{aligned}
$$

and

$8 \pi p_{t}=\frac{1}{2} e^{-\lambda}\left[\frac{1}{2}\left(v^{\prime}\right)^{2}+v^{\prime \prime}-\frac{1}{2} \lambda^{\prime} v^{\prime}+\frac{1}{r}\left(v^{\prime}-\lambda^{\prime}\right)\right]$.

In this paper, we are going to look at two particular aspects of wormhole physics, the origin of exotic matter and the enormous radial tension at any throat of moderate size.

First we need to recall that exotic matter by itself does not pose a conceptual problem, as illustrated by the Casimir effect [1]: exotic matter could be made in the laboratory. The question is whether there could ever be enough exotic matter to sustain a macroscopic traversable wormhole. In fact, some researchers consider any such wormhole solutions unphysical. It should also be emphasized that Hochberg and Visser 
have demonstrated that the wormhole throat generically violates the NEC [2]. This is an important observation since in $f(R)$ modified gravity, the throat of a wormhole can be threaded with ordinary (nonexotic) matter, while the violation of the NEC can be attributed to the higher-order curvature terms [3]. The amount of exotic matter required has also been considered, first by Visser et al. [4] and then extended by Nandi et al. [5]:

$\Omega=\int_{0}^{2 \pi} \int_{0}^{\pi} \int_{r_{0}}^{\infty}\left(\rho+p_{r}\right) \sqrt{-g} d r d \theta d \phi$.

Turning to our second problem, the high radial tension, we first need to recall that the radial tension $\tau(r)$ is the negative of the radial pressure $p_{r}(r)$. It is noted in Ref. [1] that the Einstein field equations can be rearranged to yield $\tau$ : reintroducing $c$ and $G$ for now, this is given by

$\tau(r)=\frac{b(r) / r-[r-b(r)] v^{\prime}(r)}{8 \pi G c^{-4} r^{2}}$.

From this condition it follows that the radial tension at the throat is

$\tau\left(r_{0}\right)=\frac{1}{8 \pi G c^{-4} r_{0}^{2}} \approx 5 \times 10^{41} \frac{\mathrm{dyn}}{\mathrm{cm}^{2}}\left(\frac{10 \mathrm{~m}}{r_{0}}\right)^{2}$.

In particular, for $r_{0}=3 \mathrm{~km}, \tau(r)$ has the same magnitude as the pressure at the center of a massive neutron star [1]. Attributing this outcome to exotic matter makes little sense, given that exotic matter was introduced to ensure a violation of the NEC. For example, dark matter can in principle support traversable wormholes due to the NEC violation [6,7], but the extremely low energy density cannot account for the large radial tension. The same can be said for wormholes supported by phantom dark energy [8,9]. (Equation (8) implies that $\tau\left(r_{0}\right)$ can only be small for extremely large throat sizes.) These issues will be addressed by means of the embedding theory, discussed below.

This paper is organized as follows: Sect. 2 briefly reviews the theory of embedding, including the possibility of an extra timelike dimension. Section 3 continues with the wormhole solution after introducing the coordinate transformation needed in the embedding. Section 3.3 discusses the origin of exotic matter; the amount needed can be minimized by fine-tuning two of the parameters. The flare-out condition and asymptotic flatness are discussed in Sect. 3.4. In Sect. 4, we conclude.

\section{Embedding in a five-dimensional spacetime}

Embedding theorems have a long history in the general theory of relativity, aided in large part by Campbell's theorem
[10]. According to Ref. [11], the field equations in terms of the Ricci tensor are $R_{A B}=0, A, B=0,1,2,3,4$. The resulting five-dimensional theory explains the origin of matter. More precisely, the vacuum field equations in five dimensions yield the usual Einstein field equations with matter, called the induced-matter theory $[12,13]$. The main motivation for introducing a fifth dimension is unification; our understanding of physics in four dimensions is greatly improved. Another important factor is that the extra dimension can be either spacelike or timelike. As a result, the particle-wave duality can in principle be solved because fivedimensional dynamics has two modes, depending on whether the extra dimension is spacelike or timelike [14]. So the fivedimensional relativity theory could ultimately lead to a unification of general relativity and quantum field theory.

The above discussion has shown that the embedding theory based on Campbell's theorem is an effective mathematical model. Before applying this model to wormholes, we need to introduce a refinement. The induced-matter theory is actually a non-compactified Kaluza-Klein theory of gravity. Matter is induced by a mechanism that locally embeds the four-dimensional spacetime in a Ricci-flat five-dimensional manifold [15]. This process requires only one extra dimension. Going beyond a single extra dimension requires the concept of embedding class: recall that an $n$-dimensional Riemannian space is said to be of embedding class $m$ if $m+n$ is the lowest dimension of the flat space in which the given space can be embedded. It is well known that the interior Schwarzschild solution and the Friedmann universe are of class one, while the exterior Schwarzschild solution is a Riemannian metric of class two. Because of the similarity, we can assume that a wormhole spacetime is also of class two and can therefore be embedded in a six-dimensional flat spacetime. It turns out, however, that a line element of class two can be reduced to a line element of class one, thereby making the analysis tractable. This mathematical model has proved to be extremely useful in the study of compact stellar objects [16-21]. Referring to line element (1), it is shown in Ref. [16] that a metric of class two can be reduced to a metric of class one and can therefore be embedded in the five-dimensional flat spacetime

$d s^{2}=-\left(d z^{1}\right)^{2}+\left(d z^{2}\right)^{2}+\left(d z^{3}\right)^{2}+\left(d z^{4}\right)^{2}+\left(d z^{5}\right)^{2}$

by using the coordinate transformation $z^{1}=\sqrt{K} e^{\nu / 2} \sinh \frac{t}{\sqrt{K}}$, $z^{2}=\sqrt{K} e^{\nu / 2} \cosh \frac{t}{\sqrt{K}}, z^{3}=r \sin \theta \cos \phi, z^{4}=r \sin \theta \sin \phi$, and $z^{5}=r \cos \theta$. In this paper, we are going to be more interested in an embedding space with an extra timelike dimension, discussed below. 


\section{Wormholes of embedding class one}

As noted earlier, in this paper we are primarily interested in an embedding space with an extra timelike dimension. The resulting spacetime is usually referred to as an anti-de Sitter space and is characterized by a negative cosmological constant. This is another important mathematical model that has been used in the study of many aspects of nuclear and condensed-matter physics, in particular the AdS/CFT correspondence (anti-de Sitter/comformal field theory correspondence) [22]. This theory has found its way into the study of entanglement, conjectured to be equivalent to the existence of another type of wormhole, the Einstein-Rosen bridge.

\subsection{The coordinate transformation}

Due to the extra timelike dimension, the embedding space has the form

$d s^{2}=-\left(d z^{1}\right)^{2}-\left(d z^{2}\right)^{2}+\left(d z^{3}\right)^{2}+\left(d z^{4}\right)^{2}+\left(d z^{5}\right)^{2}$.

According to Kuhfittig and Gladney [23], the coordinate transformation is $z^{1}=\sqrt{K} e^{v / 2} \sin \frac{t}{\sqrt{K}}, z^{2}=\sqrt{K} e^{v / 2} \cos \frac{t}{\sqrt{K}}$, $z^{3}=r \sin \theta \cos \phi, z^{4}=r \sin \theta \sin \phi$, and $z^{5}=r \cos \theta$. To see why, let us first list the differentials of these components:

$d z^{1}=\sqrt{K} e^{\nu / 2} \frac{v^{\prime}}{2} \sin \frac{t}{\sqrt{K}} d r+e^{v / 2} \cos \frac{t}{\sqrt{K}} d t$,

$d z^{2}=\sqrt{K} e^{\nu / 2} \frac{v^{\prime}}{2} \cos \frac{t}{\sqrt{K}} d r-e^{\nu / 2} \sin \frac{t}{\sqrt{K}} d t$,

$d z^{3}=\sin \theta \cos \phi d r+r \cos \theta \cos \phi d \theta-r \sin \theta \sin \phi d \phi$,

$d z^{4}=\sin \theta \sin \phi d r+r \cos \theta \sin \phi d \theta+r \sin \theta \cos \phi d \phi$,

and

$d z^{5}=\cos \theta d r-r \sin \theta d \theta$.

We now find that

$-\left(d z^{1}\right)^{2}-\left(d z^{2}\right)^{2}=-e^{v} d t^{2}-\frac{1}{4} K e^{v}\left(v^{\prime}\right)^{2} d r^{2}$

and

$\left(d z^{3}\right)^{2}+\left(d z^{4}\right)^{2}+\left(d z^{5}\right)^{2}=d r^{2}+r^{2}\left(d \theta^{2}+\sin ^{2} \theta d \phi^{2}\right)$.

Substituting in Eq. (11), we obtain the line element

$d s^{2}=-e^{v} d t^{2}+\left[1-\frac{1}{4} K e^{v}\left(v^{\prime}\right)^{2}\right] d r^{2}+r^{2}\left(d \theta^{2}+\sin ^{2} \theta d \phi^{2}\right)$.
Metric (17) is equivalent to metric (1) if

$e^{\lambda}=1-\frac{1}{4} K e^{v}\left(v^{\prime}\right)^{2}$,

where $K>0$ is a free parameter. Equation (2) then yields the shape function $b=b(r)$.

\subsection{The wormhole solution}

Our first step is to combine Eqs. (1) and (2) to form the more familiar line element

$d s^{2}=-e^{v(r)} d t^{2}+\frac{d r^{2}}{1-\frac{b(r)}{r}}+r^{2}\left(d \theta^{2}+\sin ^{2} \theta d \phi^{2}\right)$,

where $b=b(r)$ is obtained from Eq. (18). By the assumption of asymptotic flatness, we still have $\lim _{r \rightarrow \infty} v(r)=0$, but we also have to show that $\lim _{r \rightarrow \infty} b(r) / r=0$. To that end, we need to make one additional assumption: $v(r)$ has to be differentiable and negative, thereby approaching the $r$-axis smoothly from below. So $v^{\prime}(r)>0$ for all $r$ and $\lim _{r \rightarrow \infty} v^{\prime}(r)=0$.

According to the Introduction, our primary concerns are the violation of the NEC and the large radial tension at and near the throat. So according to Eq. (3), we need to show that $8 \pi\left(\rho+p_{r}\right)<0$. First, from Eq. (18),

$\lambda^{\prime}=\frac{-\frac{1}{4} K}{1-\frac{1}{4} K e^{v}\left(v^{\prime}\right)^{2}} e^{v} v^{\prime}\left[\left(v^{\prime}\right)^{2}+2 v^{\prime \prime}\right]$

and from the Einstein field equations,

$$
\begin{aligned}
8 \pi\left(\rho+p_{r}\right)= & e^{-\lambda} \frac{1}{r}\left(\lambda^{\prime}+v^{\prime}\right)=\frac{1}{1-\frac{1}{4} K e^{v}\left(v^{\prime}\right)^{2}} \frac{v^{\prime}}{r} \\
& \times\left[\frac{-\frac{1}{4} K e^{v}}{1-\frac{1}{4} K e^{v}\left(v^{\prime}\right)^{2}}\left[\left(v^{\prime}\right)^{2}+2 v^{\prime \prime}\right]+1\right] .
\end{aligned}
$$

Now, from Eq. (5),

$8 \pi p_{r}=\frac{1}{1-\frac{1}{4} K e^{v}\left(v^{\prime}\right)^{2}}\left(\frac{1}{r^{2}}+\frac{v^{\prime}}{r}\right)-\frac{1}{r^{2}}$.

Recalling that $\tau(r)$ is the negative of $p_{r}(r)$, we would like $p_{r}$ to be negative. Since $K$ is a free parameter, we can choose $K$ so that $\frac{1}{4} K e^{v}\left(v^{\prime}\right)^{2}>1$, making $1-\frac{1}{4} K e^{\nu}\left(v^{\prime}\right)^{2}$ negative. (We are primarily interested in the region near the throat.) For reasons that will become apparent later, we also wish to assume that $v^{\prime}(r)$ is actually quite small. Referring to Eq. (9), suppose we decide on a definite (very large) value for $\tau\left(r_{0}\right)$ for physical reasons and suppose that $v^{\prime}(r)$ has also been fixed at some small value; then the free parameter $K$ can still 
be chosen to satisfy the above conditions, while ensuring that $\left|1-\frac{1}{4} K e^{v}\left(v^{\prime}\right)^{2}\right|$ is as small as desired.

Now we can finally return to Eq. (21) to show that $8 \pi(\rho+$ $\left.p_{r}\right)$ is indeed negative. First observe that $\left(v^{\prime}\right)^{2}+2 v^{\prime \prime}$ can be made arbitrarily small near the throat "by hand." This is easier to picture by means of a simple example: if $v=-a e^{-r}$, $a>0$, then $\left(v^{\prime}\right)^{2}+\left.2 v^{\prime \prime}\right|_{r=r_{0}}=a\left(a-2 e^{r_{0}}\right) / e^{2 r_{0}} \approx 0$ if $a \approx 2 e^{r_{0}}$. It now follows at once that from Eq. (21) that

$8 \pi\left(\rho+p_{r}\right) \approx \frac{1}{1-\frac{1}{4} K e^{v}\left(\nu^{\prime}\right)^{2}} \frac{v^{\prime}}{r}<0$

since $1-\frac{1}{4} K e^{v}\left(v^{\prime}\right)^{2}<0$ and $v^{\prime}>0$. At the same time, from Eq. (22), $p_{r}\left(r_{0}\right)<0$; thus $\tau\left(r_{0}\right)$ is positive and as large as required. Finally,

$8 \pi \rho \approx \frac{1}{1-\frac{1}{4} K e^{\nu}\left(\nu^{\prime}\right)^{2}}\left(-\frac{1}{r^{2}}\right)+\frac{1}{r^{2}}>0$,

ensuring that the energy density is positive.

Given the parameters $K$ and $v$, this model requires considerable fine-tuning, which raises some questions about the stability to small perturbations. Fortunately, a sufficiently small value of $v^{\prime}\left(r_{0}\right)$ not only results in low radial tidal forces, a desirable condition for traversability, but, according to Ref. [24], it also results in a wormhole that is in stable equilibrium, based on the Tolman-Oppenheimer-Volkov equation, while remaining compatible with quantum field theory

\subsection{The total amount of exotic matter}

Returning to the induced-matter theory, since the vacuum field equations in five dimensions yield the Einstein field equations with matter, one could argue that matter is geometric in origin: what we perceive as matter is the impingement of the fifth dimension onto our spacetime; this would include exotic matter. So the amount of exotic matter may seem irrelevant, even if the amount required is large. It has always been understood, however, that the amount of exotic matter required should be kept as small as possible. Now it becomes apparent why $\nu^{\prime}$ needs to be small: we can see from Eq. (23) that for $\nu^{\prime}$ sufficiently small, $8 \pi\left(\rho+p_{r}\right)$ can be reduced as much as desired. The reason is that for any $\nu^{\prime}\left(r_{0}\right), K$ can be chosen to keep $\tau\left(r_{0}\right)$ (and hence $1-\frac{1}{4} K e^{v\left(r_{0}\right)}\left[v^{\prime}\left(r_{0}\right)\right]^{2}$ ) at the original value referred to after Eq. (22). So it follows from Eq. (7) that the amount of exotic matter can be kept to a minimum. As already noted, however, keeping the amount of exotic matter low does require considerable fine-tuning.

\subsection{Remaining conditions}

Our next task is to check the flare-out condition at the throat. Since the NEC has been violated, it ought to be true that $b^{\prime}\left(r_{0}\right)<1$, but we still need to check that this conclusion is consistent with the other assumptions. Using Eqs. (18) and (2), we can obtain by inspection

$b(r)=r\left[1-\frac{1}{1-\frac{1}{4} K e^{v(r)}\left[v^{\prime}(r)\right]^{2}}\right]+\frac{r_{0}}{1-\frac{1}{4} K e^{v\left(r_{0}\right)}\left[v^{\prime}\left(r_{0}\right)\right]^{2}}$,

ensuring that $b\left(r_{0}\right)=r_{0}$. Since $\left(v^{\prime}\right)^{2}+2 v^{\prime \prime}$ can be made arbitrarily small near the throat, it follows that

$b^{\prime}\left(r_{0}\right) \approx 1-\frac{1}{1-\frac{1}{4} K e^{v}\left(v^{\prime}\right)^{2}}<1$

due to the crucial assumption that $v^{\prime}$ is sufficiently small. Finally,

$\frac{b(r)}{r}=1-\frac{1}{1-\frac{1}{4} K e^{v}\left(v^{\prime}\right)^{2}} \rightarrow 0$

since $v^{\prime}(r) \rightarrow 0$. So the spacetime is asymptotically flat.

\section{Summary}

Embedding theorems have continued to be a topic of interest in the general theory of relativity, as exemplified by the induced-matter theory. This paper deals with spacetimes of embedding class one, i.e., spacetimes that can be embedded in a five-dimensional flat spacetime. This model has proved to be extremely useful in the study of compact stellar objects. To address the issues in this paper, we assume that the fifth dimension in the embedding space is timelike, a model that is consistent with Einstein's theory. It is shown that the problematical exotic matter needed to sustain a Morris-Thorne wormhole originates in the higher-dimensional flat spacetime, thereby becoming part of the induced-matter theory. A critical assumption is that the redshift function $v(r)$ is negative with $\nu^{\prime}(r)>0$ near $r=r_{0}$ and sufficiently small, thereby keeping the amount of exotic matter to a minimum. Finally, thanks to the free parameter $K$ and the properties of $v(r)$, the embedding theory can account for the other problematical issue, the enormous radial tension at the throat of a Morris-Thorne wormhole.

The dependence on the parameters $K$ and $v$ has resulted in the need for considerable fine-tuning, a problem not easily avoided. It is shown in Ref. [25] that a Morris-Thorne wormhole held open by a small amount of exotic matter can be made compatible with quantum field theory only through extreme fine-tuning of the metric coefficients. This problem may be avoidable in $f(R)$ modified gravity, but this approach does not explain the large radial tension. That issue 
has been addressed in Ref. [26], however: it is shown that the large radial tension can be accounted for via noncommutative geometry, an offshoot of string theory, or by the existence of a small extra spatial dimension. The latter complements the conclusions in the present paper.

Data Availability Statement This manuscript has no associated data or the data will not be deposited. [Authors' comment: This paper is purely theoretical. (there are no experimental data.).]

Open Access This article is licensed under a Creative Commons Attribution 4.0 International License, which permits use, sharing, adaptation, distribution and reproduction in any medium or format, as long as you give appropriate credit to the original author(s) and the source, provide a link to the Creative Commons licence, and indicate if changes were made. The images or other third party material in this article are included in the article's Creative Commons licence, unless indicated otherwise in a credit line to the material. If material is not included in the article's Creative Commons licence and your intended use is not permitted by statutory regulation or exceeds the permitted use, you will need to obtain permission directly from the copyright holder. To view a copy of this licence, visit http://creativecomm ons.org/licenses/by/4.0/.

Funded by SCOAP ${ }^{3}$.

\section{References}

1. M.S. Morris, K.S. Thorne, Am. J. Phys. 56, 395 (1988)

2. D. Hochberg, M. Visser, Phys. Rev. D 56, 4745 (1997)

3. F.S.N. Lobo, M.A. Oliveira, Phys. Rev. D 80, 104012 (2009)

4. M. Visser, S. Kar, N. Dadhich, Phys. Rev. Lett. 90, 201102 (2003)

5. K.K. Nandi, Y.-Z. Zhang, K.B. Vijaya Kumar, Phys. Rev. D 70, 127503 (2004)

6. F. Rahaman, P. Salucci, P.K.F. Kuhfittig, S. Ray, M. Rahaman, Ann. Phys. 350, 561 (2014)

7. Z. Xu, M. Tang, G. Cao, S.-N. Zhang, Eur. Phys. J. C 80, 70 (2020)
8. S.V. Sushkov, Phys. Rev. D 71, 043520 (2005)

9. F.S.N. Lobo, Phys. Rev. D 71, 084011 (2005)

10. J. Campbell, A Course on Differential Geometry (Clarenden, Oxford, 1926)

11. P.S. Wesson, Int. J. Mod. Phys. D 24, 1530001 (2015)

12. P.S. Wesson, J. Ponce de Leon, J. Math. Phys. 33, 3883 (1992)

13. S.S. Seahra, P.S. Wesson, Class. Quantum Gravity 20, 1321 (2003)

14. P.S. Wesson, Phys. Lett. B 706, 1 (2011)

15. J.B. Fonseca-Neto, C. Romero, F. Dahia, Braz. J. Phys. 35, 1067 (2005)

16. S.K. Maurya, M. Govender, Eur. Phys. J. C 77, 347 (2017)

17. S.K. Maurya, S.D. Maharaj, Eur. Phys. J. C 77, 328 (2017)

18. S.K. Maurya, B.S. Ratanpal, M. Govender, Ann. Phys. 382, 36 (2017)

19. S.K. Maurya, Y.K. Gupta, S. Ray, D. Deb, Eur. Phys. J. C 77, 45 (2017)

20. S.K. Maurya, Y.K. Gupta, S. Ray, D. Deb, Eur. Phys. J. C 76, 693 (2016)

21. S.K. Maurya, D. Deb, S. Ray, P.K.F. Kuhfittig, Int. J. Mod. Phys. D 28, 1950116 (2019)

22. J. Maldacena, Adv. Theor. Math. Phys. 2, 231 (1998)

23. P.K.F. Kuhfittig, V.D. Gladney, Adv. Stud. Theor. Phys. 12, 233 (2018)

24. P.K.F. Kuhfittig, Fund. J. Mod. Phys. 14, 23 (2020)

25. P.K.F. Kuhfittig, arXiv:0908.4233 [gr-qc]

26. P.K.F. Kuhfittig, Eur. Phys. J. Plus 135, 50 (2020) 